\title{
Collective Response in DNA-Stabilized Silver Cluster Assemblies from First-Principles Simulations
}

\author{
Polina G. Lisinetskaya* and Roland Mitrić* \\ Institut für Physikalische und Theoretische Chemie, Universität Würzburg, D-97074 \\ Würzburg, Germany \\ E-mail: polina.lisinetskaya@uni-wuerzburg.de; roland.mitric@uni-wuerzburg.de
}




\begin{abstract}
We investigate fluorescence resonant energy transfer and concurrent electron dynamics in a pair of DNA-stabilized silver clusters. For this purpose we introduce a methodology for the simulation of collective opto-electronic properties of coupled molecular aggregates starting from first-principles quantum chemistry, which can be further applied to a broad range of coupled molecular systems to study their electrooptical response. Our simulations reveal the existence of a low-energy coupled excitonic states, which enable ultrafast energy transport between subunits, and give an insight into the origin of the fluorescence signal in coupled DNA-stabilized silver clusters, that have been recently experimentally detected. Hence we demonstrate the possibility to construct an ultra-small energy transmission lines and optical converters based on these hybrid molecular systems.
\end{abstract}

\title{
Graphical TOC Entry
}

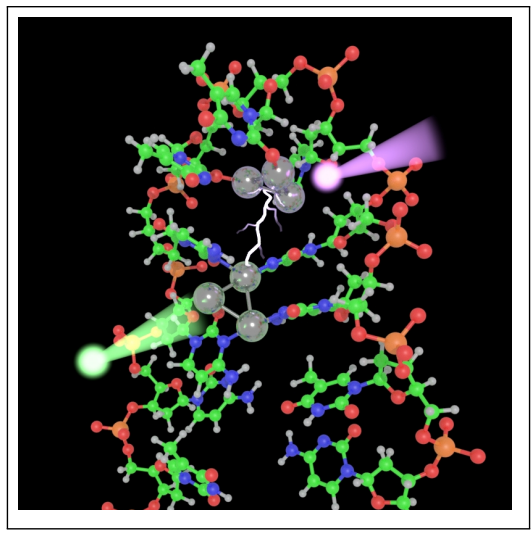


Ligand-stabilized silver clusters represent promising building blocks for novel ultra-small optical and electronic devices. ${ }^{1-8}$ The sphere of their proven applications is impressively broad ranging from biosensing ${ }^{9-11}$ to photovoltaics. ${ }^{12,13}$ Especially one of the hottest topics of modern cluster science is represented by DNA-stabilized few-atom sliver clusters. ${ }^{14}$ Experimentally, significant progress has been achieved in synthesis of these highly fluorescent and photo-stable complexes ${ }^{15,16}$ demonstrating the possibility to create DNA-embedded silver clusters with absorption and emission spectra tunable in the whole visible range and even beyond it. ${ }^{17-19}$ Very recent experimental advances allowed to shed light on the structures of these hybrid systems as well. ${ }^{20,21}$

For theory these systems represent considerable challenge due to their size. Indeed, the simulations show, that in order to reliably reproduce the experimental results, accurate quantum-chemical calculations are required, ${ }^{22,23}$ which include not only a silver cluster core, but also adjacent nucleobases, because the latter can dramatically change the optical properties of the cluster. ${ }^{24}$ In addition, proper taking into account of the solvent environment is mandatory as well. ${ }^{25}$ Currently, significant progress has been made in understanding of the structure and charge distributions in individual DNA-stabilized silver clusters employing force-field molecular dynamics simulations, ${ }^{26,27}$ as well as coupled quantummechanical/molecular-mechanical (QM/MM) methods, ${ }^{22,28}$ and density functional theory (DFT). ${ }^{23,24,29-31}$ In contrast, the collective properties of DNA-stabilized silver cluster assemblies, although experimentally proven, ${ }^{16}$ still remain undeservedly beyond the scope of the up-to-date theoretical research. However, such aggregates could provide unique possibility to construct nanosized optoelectronic devices based on spatially organized arrays of noblemetal clusters serving, for instance, as ultra-small energy and charge transmission lines. ${ }^{32-34}$ Therefore, an efficient theoretical method for a realistic description of exciton and charge propagation in such systems under the action of an external driving electric field is highly desired.

In this Letter, we introduce a methodology for modeling of the collective optical response 
and laser-driven electron dynamics in coupled cluster-organic aggregates aiming to pave the avenue for the design of novel quantum materials. Here, we apply our methodology to simulate resonant energy transfer process in an aggregate of DNA-stabilized silver clusters. This effect has already been experimentally demonstrated, ${ }^{16}$ but to date, a corresponding theoretical model has not been yet established.

Briefly, the proposed approach combines the first-principles description of the electronic structure of individual constituents with fully quantum-mechanical propagation of the Liouvillevon Neumann equation under the action of an external laser field. This allows to describe exciton dynamics in a coupled aggregate. Further, methods of classical electrodynamics are employed to simulate the response of the aggregate including fluorescence and local electric field distribution (see Supporting Information for details).

The simplicity and power of the described approach can be demonstrated on the example of a hybrid silver-DNA system consisting of two DNA-stabilized $\mathrm{Ag}_{3}^{+}$clusters, namely $\mathrm{Ag}_{3}^{+} \mathrm{CT}_{2}$ and $\mathrm{Ag}_{3}^{+} \mathrm{C}_{4}$ (C and $\mathrm{T}$ stand for cytosine and thymine, respectively) shown in Fig. 1 (a) and (b). The geometrical structures of the silver-DNA subunits have been determined
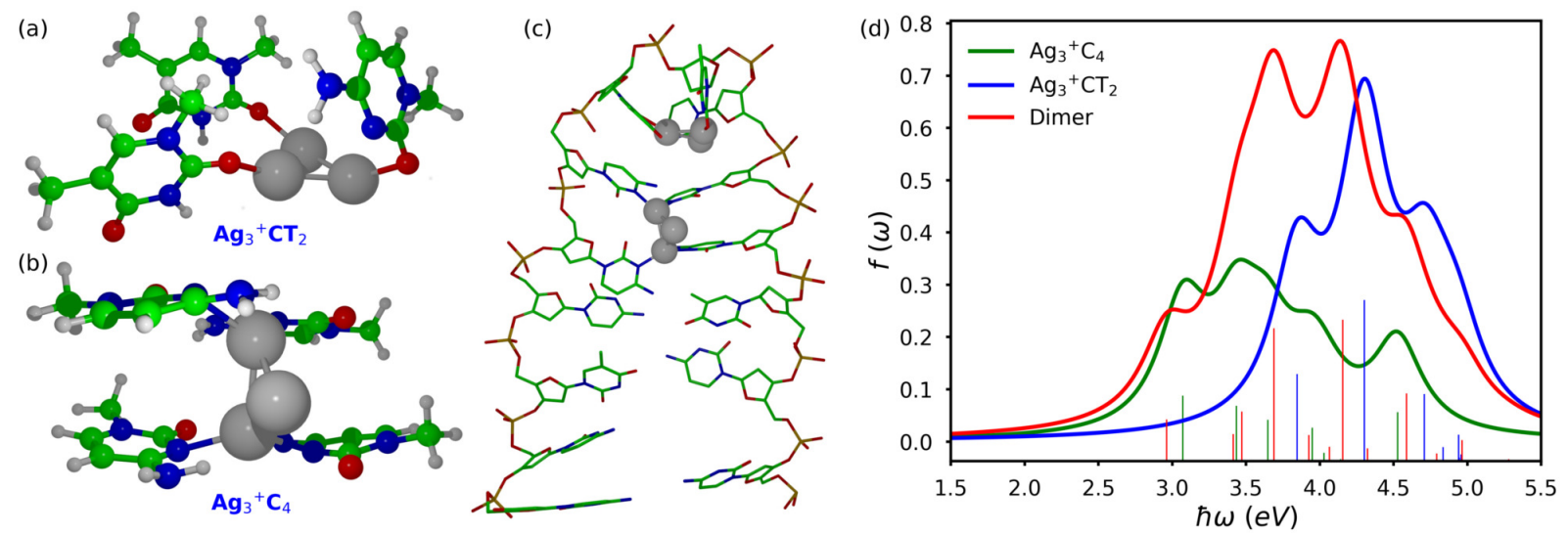

Figure 1: (a)-(b) Ground-state nuclear geometries of (a) $\mathrm{Ag}_{3}^{+} \mathrm{CT}_{2}$, and (b) $\mathrm{Ag}_{3}^{+} \mathrm{C}_{4}$ clusters; (c) schematic representation of a DNA hairpin structure with the two clusters embedded in it; (d) absorption spectra of the individual clusters and the dimer structure.

previously by Ramazanov et al. employing accurate DFT-QM/MM molecular dynamics simulations. ${ }^{22}$ The DNA-stabilized $\mathrm{Ag}_{3}^{+}$clusters, although not the dominant experimentally 
detected structures of such type so far, are confirmed by other experiments ${ }^{35}$ and represent smallest stable DNA-encapsulated silver clusters, which facilitates the simulations. Therefore we have used these structures as starting points in our calculations.

These fluorescent DNA-stabilized silver clusters were experimentally observed under the same conditions at different binding sites of the same DNA-hairpin structure. ${ }^{22}$ Therefore, we can assume, that by adjusting of the experimental conditions and the DNA sequence, the two clusters may be brought together in a single hairpin as schematically shown in Fig. 1 (c). The $\mathrm{Ag}_{3}^{+} \mathrm{CT}_{2}$ subunit is located on top of the hairpin followed by the $\mathrm{Ag}_{3}^{+} \mathrm{C}_{4}$ subunit, which is in agreement with the results of the simulations. ${ }^{22}$ We would like to emphasize, that experimental realization of such coupled cluster dimer can be very challenging and may lead to serious distortion of the clusters' geometry. However, the developed methodology will be still applicable to such system and the collective behavior described below should be detectable if the clusters are close enough. To date, only a few cluster aggregates have been experimentally demonstrated, ${ }^{16,36}$ where each cluster was individually encapsulated in a DNA strand, which led to the inter-cluster distance of several $\mathrm{nm}$ and a weak coupling between them. Since the investigation of a cluster aggregate formation lies beyond the scope of the current study and represents a challenging problem on its own, we construct the dimer assuming that the silver cores are bound to neighboring nucleobases. Since the nucleobases are connected via the sugar backbone, the relative position and orientation of the silver cores is nearly fixed. The distance between the centers of the silver cores is estimated to be approximately $5 \AA$, the mutual orientation of the transition dipole moments is provided in Supporting Information.

The field-free dimer Hamiltonian allows to extract the absorption spectrum of this aggregate. The spectrum is presented in Fig. 1 (d) together with the absorption spectra of the individual subunits, simulated employing the linear response time-dependent densityfunctional theory (TDDFT). ${ }^{37}$ The difference between the absorption spectra of two individual Ag-DNA clusters is due to their excited state electronic structure. In the $\mathrm{Ag}_{3}^{+} \mathrm{C}_{4}$ silver 
atoms are bound to the endocyclic nitrogen, which enables significant charge transfer from the silver core to the cytosine, while in the $\mathrm{Ag}_{3}^{+} \mathrm{CT}_{2}$ silver atoms interact mainly with oxygen and comparable charge transfer is not observed. The comparison of the dimer spectrum to the results of the direct TDDFT calculations is very convincing (see Supporting Information, Fig. S1) and confirms the validity of our approximate methodology. It is evident, that the dimer spectrum can be roughly represented by the sum of the absorption spectra of individual subunits, suggesting that the optical properties of the two DNA-embedded silver clusters do not change dramatically, even when they are connected to neighboring nucleobases. This spectral feature is confirmed by analyzing the dimer wave-functions corresponding to the low-lying excited states (cf. Table S1). In all dimer excited states below $4 \mathrm{eV}$ excitons are mainly (more than 90\%) localized on one of the subunits.

In order to simulate the emission spectrum of the silver-DNA dimer, the electron dynamics induced in the aggregate by a linearly polarized laser pulse was calculated. The temporal profile of the pulse was assumed to have a Gaussian shape with a full width at half maximum equal to 60 fs and a peak electric field strength of $5 \cdot 10^{9} \mathrm{~V} / \mathrm{m}$ (corresponding to the peak power of $3.3 \cdot 10^{12} \mathrm{~W} / \mathrm{cm}^{2}$ ). The central frequency was varied to cover the range of 2.9-4.0 $\mathrm{eV}$, to which the two lowest absorption bands of the aggregate belong. For each excitation frequency, the corresponding emission spectrum was obtained.

The results are combined in the 2D map presented in Fig. 2 (a). The main fluorescence signal is located on the diagonal, demonstrating that the aggregate emits photons with the same energy as it absorbs. This is natural, since there is no nuclear relaxation included in the model and therefore no Stokes shift between the absorbed and emitted wavelengths is introduced. This assumption is valid since the time scale of the dimer response studied here (sub-picosecond regime) is much shorter than the rearrangement of heavy silver atoms (typically several picoseconds ${ }^{38}$ ). The four pronounced peaks along the diagonal in the dimer spectrum correspond to the excitation energies of the lowest excited electronic states of the coupled system (see Table S1). However, one off-diagonal peak is clearly seen in the aggregate 

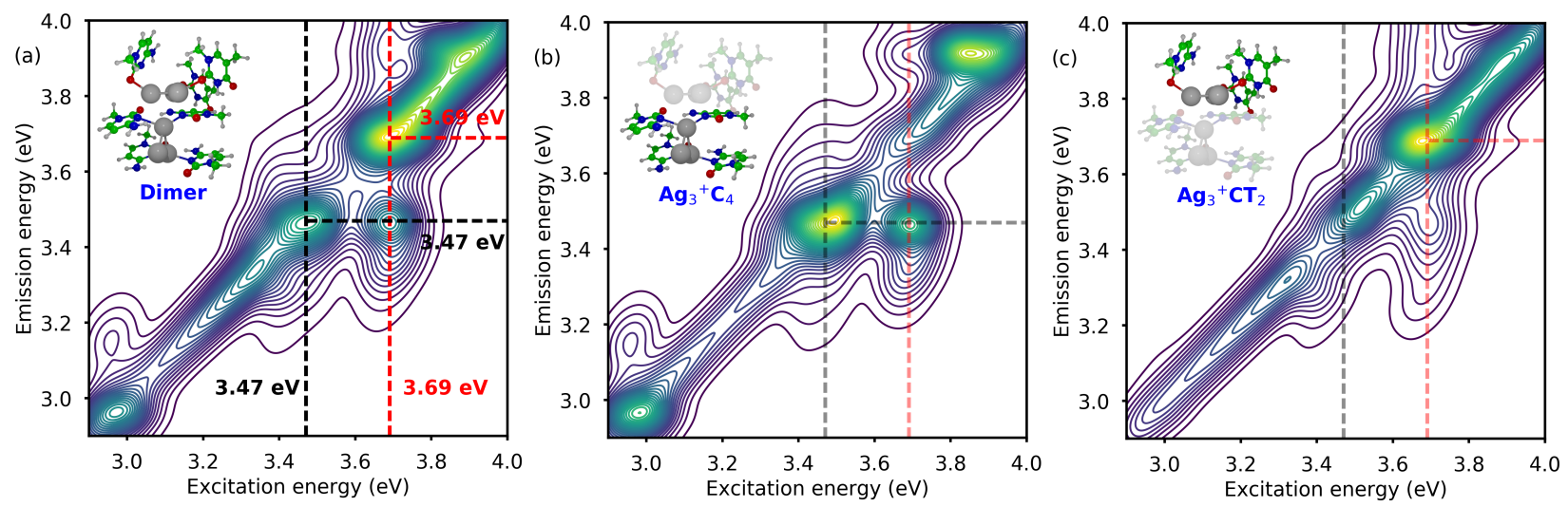

Figure 2: Fluorescence spectra as function of the excitation energy. (a) Signal from the coupled dimer structure, (b)-(c) contributions to the dimer signal arising from the $\mathrm{Ag}_{3}^{+} \mathrm{C}_{4}$ (b) and $\mathrm{Ag}_{3}^{+} \mathrm{CT}_{2}$ (c) clusters. Dashed horizontal and vertical lines correspond to $3.47 \mathrm{eV}$ (black) and $3.69 \mathrm{eV}$ (red) energies. The insets in (a)-(c) present the structure, from which the corresponding signal arises.

spectrum at the excitation energy of $3.69 \mathrm{eV}$, which indicates energy transfer between two cluster subunits.

Indeed, first we note that in the excited state of the dimer corresponding to $3.69 \mathrm{eV}$ the exciton is mainly localized on the $\mathrm{Ag}_{3}^{+} \mathrm{CT}_{2}$ subunit (cf. Table S1). Second, employing the reduced density matrix approach, the optical response of the dimer can be separated into contributions arising due to the individual subunits. In Fig. 2 (b) and (c) the inputs to the total fluorescence signal from each particular cluster are presented. It is seen that at the excitation energy of $3.69 \mathrm{eV}$ the two peaks in the dimer emission spectrum can be attributed to the signal coming from two different clusters, namely, the peak at $3.47 \mathrm{eV}$ from $\mathrm{Ag}_{3}^{+} \mathrm{C}_{4}$ cluster and the one at $3.69 \mathrm{eV}$ from $\mathrm{Ag}_{3}^{+} \mathrm{CT}_{2}$, respectively. Therefore, the off-diagonal peak in the fluorescence excitation spectrum represents resonant energy transfer from the $\mathrm{Ag}_{3}^{+} \mathrm{CT}_{2}$ subunit to the $\mathrm{Ag}_{3}^{+} \mathrm{C}_{4}$, which as a result emits radiation with different wavelengths to the incident one. This peak is analogous to the Förster resonant energy transfer (FRET) feature, which is experimentally observed in a dimer of larger DNA-stabilized silver clusters ${ }^{16}$ or in an Ag-DNA coupled to an organic dye. ${ }^{39}$

The underlying mechanism can be understood upon the investigation of the electron population dynamics induced in the dimer by the laser pulse of $3.69 \mathrm{eV}$ photon energy 
(shown in Fig. S3). In order to reveal the corresponding dynamical processes occurring in the single clusters, the reduced density matrix approach was employed to partition it into population dynamics in each subunit individually, as presented in Fig. 3 (a), (b). The

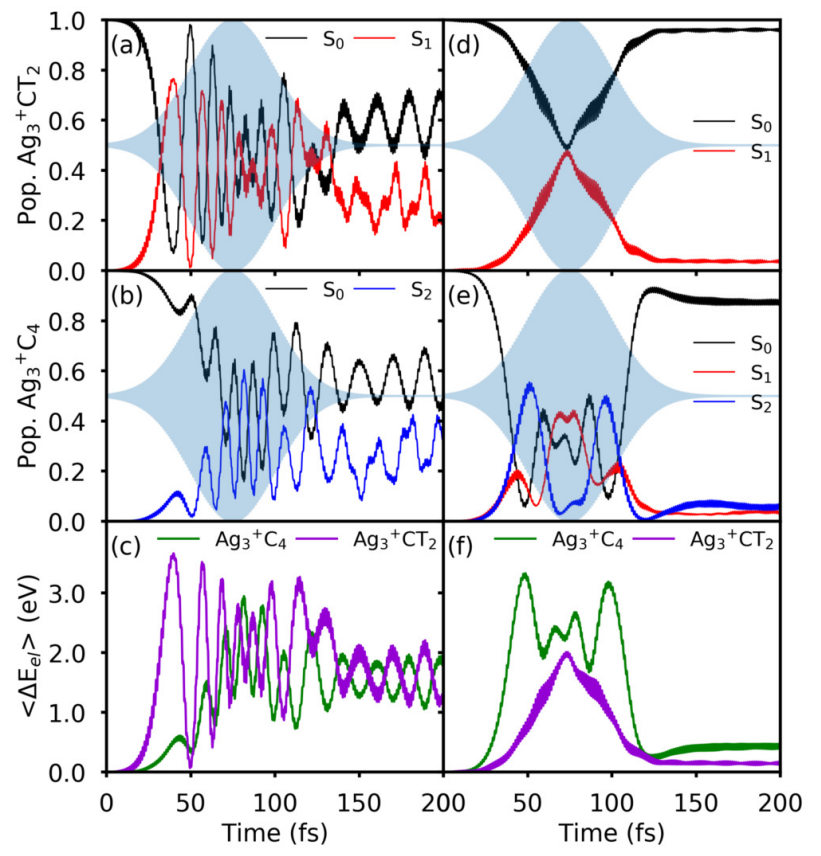

Figure 3: (left column) Electron population dynamics excited by a $3.69 \mathrm{eV}$ laser pulse in (a) $\mathrm{Ag}_{3}^{+} \mathrm{CT}_{2}$ and (b) $\mathrm{Ag}_{3}^{+} \mathrm{C}_{4}$ clusters. As the shaded blue area the driving laser pulse is schematically presented. (c) Expectation values of the single cluster electron energies. (right column) The same as in the left column, but in case of excitation with a $3.47 \mathrm{eV}$ pulse.

simulations demonstrate that at $3.69 \mathrm{eV}$ photon energy the laser pulse first causes population transfer to the $\mathrm{S}_{1}$ state of $\mathrm{Ag}_{3}^{+} \mathrm{CT}_{2}$ and only when this state is significantly populated, electron population transfer to the $\mathrm{S}_{2}$ state of $\mathrm{Ag}_{3}^{+} \mathrm{C}_{4}$ occurs. During the laser pulse action the electron populations of $\mathrm{Ag}_{3}^{+} \mathrm{CT}_{2}$ perform several cycles of Rabi-like oscillations, each cycle leading to an increase of the amount of excited state population in $\mathrm{Ag}_{3}^{+} \mathrm{C}_{4}$. After $140 \mathrm{fs}$, when the driving pulse ceases, the ground electronic states of both clusters are nearly equally depopulated. Unlike for isolated clusters, the electron population continues to oscillate in counter-phase even when no external perturbation is present. This effect reflects the coupled nature of the excited state, induced in the Ag-DNA dimer by the laser field at the given frequency. The clear fingerprints of coherent energy transfer according to 
the Förster mechanism can be seen in the single-cluster electron energy dynamics presented in Fig. 3 (c), which demonstrates coherent out-of-phase oscillations of the electron energy between the clusters after the laser pulse ceases.

Substantially different electron population dynamics is induced in the dimer by the 3.47 eV laser pulse (see Fig. S4). In the single-cluster picture (Fig. 3 (d) and (e)) the external laser pulse promotes the electron population of $\mathrm{Ag}_{3}^{+} \mathrm{C}_{4}$ simultaneously to the $\mathrm{S}_{1}$ and $\mathrm{S}_{2}$ states with subsequent population transfer to the $\mathrm{S}_{1}$ state of the $\mathrm{Ag}_{3}^{+} \mathrm{CT}_{2}$ cluster. However, no coupled excited state is produced by the laser pulse with $3.47 \mathrm{eV}$ photon energy and no energy transfer between the clusters is observed (see Fig. 3 (f)).

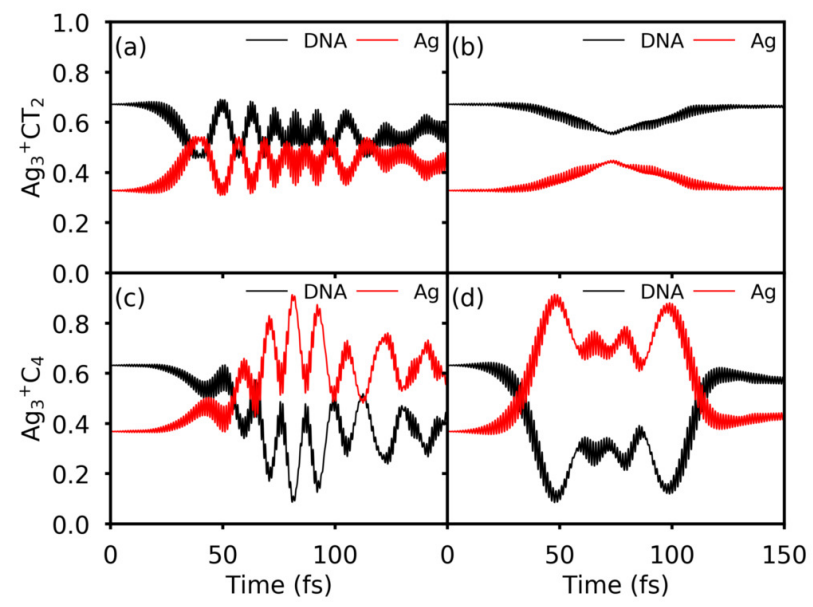

Figure 4: Time-dependent partial charges, localized on the (red lines) silver and (black lines) DNA parts of the (a), (b) $\mathrm{Ag}_{3}^{+} \mathrm{CT}_{2}$ and (c), (d) $\mathrm{Ag}_{3}^{+} \mathrm{C}_{4}$ clusters. The charge dynamics was induced by (a), (c) $3.69 \mathrm{eV}$, and (b), (d) $3.47 \mathrm{eV}$ pulses.

Finally, we investigate the dynamics of charge transfer between silver core and DNA nucleobases occurring within two subunits upon photo-excitation. The time-dependent partial charges localized on the silver core and DNA-part of each single subunit were simulated as expectation values of the charge operators $\hat{\mathrm{q}}_{I, a}$. In the $\mathrm{Ag}_{3}^{+} \mathrm{C}_{4}$ cluster, noticeable charge transfer from Ag to DNA is observed at both excitation pulse energies (see Fig. 4 (c), (d)), while no significant charge transfer between silver and DNA parts of $\mathrm{Ag}_{3}^{+} \mathrm{CT}_{2}$ occurs (cf. Fig. 4 (a), (b)). These charge dynamics reflect the character of the excited states of the two clusters, with exciton mainly localized on the silver core in $\mathrm{Ag}_{3}^{+} \mathrm{CT}_{2}$ and more delocalized in 
$\mathrm{Ag}_{3}^{+} \mathrm{C}_{4}$.

In the ground state the partial charge of the silver core is 0.37 in $\mathrm{Ag}_{3}^{+} \mathrm{C}_{4}$ and 0.33 in $\mathrm{Ag}_{3}^{+} \mathrm{CT}_{2}$ complexes. Interestingly, for an $\mathrm{Ag}^{+}$ion bridging two cytosine molecules the partial charge was previously determined as $0.38272,{ }^{27}$ which is very close to the partial charge of the $\mathrm{Ag}_{3}^{+}$cluster in $\mathrm{Ag}_{3}^{+} \mathrm{C}_{4}$ in our simulations and seems to be characteristic for silverbridged $\mathrm{C}-\mathrm{Ag}_{n}^{+}-\mathrm{C}$ aggregates. Upon photo-excitation, the partial charge on the silver core in $\mathrm{Ag}_{3}^{+} \mathrm{C}_{4}$ noticeably increases, reaching at some instants of time values close to 1.0. This effect is observed for any frequency of the driving laser pulse resonant to an excited state of the dimer. Figs. 4 (c) and (d) demonstrate that this type of charge transfer dynamics is observed at 3.47 , as well as $3.69 \mathrm{eV}$ laser pulses, although the electron population dynamics are significantly different. At the same time, the charge transfer within the $\mathrm{Ag}_{3}^{+} \mathrm{CT}_{2}$ cluster is much less pronounced for both investigated laser pulses. This effect demonstrates the impact of the adjacent nucleobases on the charge transfer dynamics in DNA-stabilized silver clusters and once again underlines the importance of proper inclusion of organic templates or ligand molecules in the simulations of optical and electronic properties of such noble-metal clusters.

In conclusion, we have developed a methodology to investigate the coupled optical response in aggregates of ligand-stabilized silver cluster assemblies. The approach is general and is not only limited to DNA-stabilized silver clusters, but is also applicable to clusters, biomolecules or hybrid systems with sizes comparable or larger than the distance between the interacting subunits, which is essential for the investigation of energy and charge transport properties of molecular or ligand-protected metal cluster aggregates, as well as of cluster arrays deposited on an organic template for both fundamental and application-oriented purposes. The proposed methodology represents a practical tool for the design of novel nanooptical devices based on ultra-small metal clusters and for the simulation of their functionality. Since the driving electric field is directly included in the computational scheme, the method can be straightforwardly coupled with a genetic algorithm for the simulation of 
optimal control experiments.

Using the developed theoretical method an energy transfer dynamics in a pair of $\mathrm{Ag}_{3}^{+}$ clusters embedded in DNA was simulated, confirming that the nucleobase environment can dramatically affect not only the absorption and emission, but also the charge transfer dynamics in the cluster. It was demonstrated that in such hybrid systems coupled excited states can be created that allow ultrafast energy transport within the aggregate even after the driving electric field has ceased. The origin of the absorption and fluorescence signal was revealed confirming the energy transfer between silver cluster subunits. In general, the simulations demonstrate novel functionalities of the Ag-DNA aggregates including energy transport and optical conversion properties, and will hopefully inspire extensive experimental research in this field.

\section{Computational methods}

The optical properties of the individual Ag-DNA subunits were determined using linearresponse TDDFT with Coulomb-attenuated gradient-corrected CAM-B3LYP functional ${ }^{40}$ and quadruple-zeta valence plus polarization (def2-QZVPP) basis sets for all atoms ${ }^{41}$ as implemented in the program code Gaussian09 Rev. D.01. ${ }^{42}$ The results of these calculations were also further used as prerequisites for simulation of the absorption spectrum of the aggregate, as well as the exciton and charge dynamics, and the emission spectra. Detailed description of the methodology is provided in Supporting Information.

\section{Acknowledgement}

The authors gratefully acknowledge financial support of the European Research Council in the frame of ERC Consolidator Grant DYNAMO (Grant No. 646737). 


\section{Supporting Information Available}

Theoretical method with derivation of the working equations, excited state properties of the single clusters and the dimer, optical response of the individual clusters. This material is available free of charge via the Internet at http://pubs.acs.org.

\section{References}

(1) Peyser, L. A.; Vinson, A. E.; Bartko, A. P.; Dickson, R. M. Photoactivated Fluorescence from Individual Silver Nanoclusters. Science 2001, 291, 103-106.

(2) Zheng, J.; Zhang, C. W.; Dickson, R. M. Highly Fluorescent, Water-Soluble, SizeTunable Gold Quantum Dots. Phys. Rev. Lett. 2004, 93, 077402.

(3) Johnson, G. E.; Mitrić, R.; Bonačić-Koutecký, V.; Castleman, J. A. W. Clusters as Model Systems for Investigating Nanoscale Oxidation Catalysis. Chem. Phys. Lett. 2009, 475, 1-9.

(4) Benson, O. Assembly of Hybrid Photonic Architectures from Nanophotonic Constituents. Nature 2011, 480, 193-199.

(5) Gwinn, E.; Schultz, D.; Copp, S. M.; Swasey, S. DNA-Protected Silver Clusters for Nanophotonics. Nanomaterials 2015, 5, 180-207.

(6) Cuerva, M.; García-Fandiño, R.; Vázquez-Vázquez, C.; López-Quintela, M. A.; Montenegro, J.; Granja, J. R. Self-Assembly of Silver Metal Clusters of Small Atomicity on Cyclic Peptide Nanotubes. ACS Nano 2015, 9, 10834-10843.

(7) Röhr, M. I. S.; Lisinetskaya, P. G.; Mitrić, R. Excitonic Properties of Ordered Metal Nanocluster Arrays: 2D Silver Clusters at Multiporphyrin Templates. J. Phys. Chem. A 2016, 120, 4465-4472. 
(8) Lisinetskaya, P. G.; Röhr, M. I. S.; Mitrić, R. First-Principles Simulation of Light Propagation and Exciton Dynamics in Metal Cluster Nanostructures. Appl. Phys. B 2016, 122, 175.

(9) Wu, X.; He, X.; Wang, K.; Xie, C.; Zhou, B.; Qing, Z. Ultrasmall Near-Infrared Gold Nanoclusters for Tumor Fluorescence Imaging in Vivo. Nanoscale 2010, 2, 2244-2249.

(10) Shang, L.; Dong, S.; Nienhaus, G. U. Ultra-small Fluorescent Metal Nanoclusters: Synthesis and Biological Applications. Nano Today 2011, 6, 401-418.

(11) Xiao, Y.; Shu, F.; Wong, K.-Y.; Liu, Z. Förster Resonance Energy Transfer-Based Biosensing Platform with Ultrasmall Silver Nanoclusters as Energy Acceptors. Anal. Chem. 2013, 85, 8493-8497.

(12) Hasobe, T.; Imahori, H.; Kamat, P. V.; Ahn, T. K.; Kim, S. K.; Kim, D.; Fujimoto, A.; Hirakawa, T.; Fukuzumi, S. Photovoltaic Cells Using Composite Nanoclusters of Porphyrins and Fullerenes with Gold Nanoparticles. J. Am. Chem. Soc. 2005, 127, 12161228.

(13) Chen, Y.-S.; Choi, H.; Kamat, P. V. Metal-Cluster-Sensitized Solar Cells. A New Class of Thiolated Gold Sensitizers Delivering Efficiency Greater Than 2\%. J. Am. Chem. Soc. 2013, 135, 8822-8825.

(14) Petty, J. T.; Zheng, J.; Hud, N. V.; Dickson, R. M. DNA-Templated Ag Nanocluster Formation. J. Am. Chem. Soc. 2004, 126, 5207-5212.

(15) Vosch, T.; Antoku, Y.; Hsiang, J.; Richards, C.; Gonzalez, J.; Dickson, R. Strongly Emissive Individual DNA-encapsulated Ag Nanoclusters as Single-molecule Fluorophores. Proc. Natl. Acad. Sci. U.S.A. 2007, 104, 12616-12621.

(16) Schultz, D.; Copp, S. M.; Markešević, N.; Gardner, K.; Oemrawsingh, S. S. R.; Bouwmeester, D.; Gwinn, E. Dual-Color Nanoscale Assemblies of Structurally Stable, 
Few-Atom Silver Clusters, As Reported by Fluorescence Resonance Energy Transfer. ACS Nano 2013, 7, 9798-9807.

(17) Sharma, J.; Yeh, H.-C.; Yoo, H.; Werner, J. H.; Martinez, J. S. A Complementary Palette of Fluorescent Silver Nanoclusters. Chem. Commun. 2010, 46, 3280-3282.

(18) Copp, S. M.; Schultz, D.; Swasey, S.; Pavlovich, J.; Debord, M.; Chiu, A.; Olsson, K.; Gwinn, E. Magic Numbers in DNA-Stabilized Fluorescent Silver Clusters Lead to Magic Colors. J. Phys. Chem. Lett. 2014, 5, 959-963.

(19) Swasey, S. M.; Copp, S. M.; Nicholson, H. C.; Gorovits, A.; Bogdanov, P.; Gwinn, E. G. High Throughput Near Infrared Screening Discovers DNA-Templated Silver Clusters with Peak Bluorescence Beyond 950 nm. Nanoscale 2018, 10, 19701-19705.

(20) Huard, D. J. E.; Demissie, A.; Kim, D.; Lewis, D.; Dickson, R. M.; Petty, J. T.; Lieberman, R. L. Atomic Structure of a Fluorescent Ag8 Cluster Templated by a Multistranded DNA Scaffold. J. Am. Chem. Soc. 2019, 141, 11465-11470.

(21) Cerretani, C.; Kanazawa, H.; Vosch, T.; Kondo, J. Crystal Structure of a NIR-Emitting DNA-Stabilized Ag16 Nanocluster. Angew. Chem. 2019, doi: 10.1002/ange.201906766.

(22) Ramazanov, R. R.; Sych, T. S.; Reveguk, Z. V.; Maksimov, D. A.; Vdovichev, A. A.; Kononov, A. I. Ag-DNA Emitter: Metal Nanorod or Supramolecular Complex? J. Phys. Chem. Lett. 2016, 7, 3560-3566.

(23) Bonačić-Koutecký, V.; Perić, M.; Sanader, Ž. Why Do Silver Trimers Intercalated in DNA Exhibit Unique Nonlinear Properties That Are Promising for Applications? J. Phys. Chem. Lett. 2018, 9, 2584-2589.

(24) de Carvalho, L. C.; Silveira, O. J.; Longuinhos, R.; Nunes, R. W.; Alexandre, S. S. Optical Absorption in Complexes of Abasic DNA with Noble-Metal Nanoclusters by First Principles Calculations. Phys. Chem. Chem. Phys. 2019, 21, 1260-1270. 
(25) Cerretani, C.; Vosch, T. Switchable Dual-Emissive DNA-Stabilized Silver Nanoclusters. ACS Omega 2019, 4, 78957902.

(26) Staelens, N.; Leherte, L.; Champagne, B.; Vercauteren, D. P. Modeling of Structural, Energetic, and Dynamic Properties of Few-Atom Silver Clusters Embedded in Polynucleotide Strands by Using Molecular Dynamics. ChemPhysChem 2014, 16, 360-369.

(27) Chen, X.; Karpenko, A.; Lopez-Acevedo, O. Silver-Mediated Double Helix: Structural Parameters for a Robust DNA Building Block. ACS Omega 2017, 2, 7343-7348.

(28) Makkonen, E.; Rinke, P.; Lopez-Acevedo, O.; Chen, X. Optical Properties of SilverMediated DNA from Molecular Dynamics and Time Dependent Density Functional Theory. Int. J. Mol. Sci. 2018, 19, 2346.

(29) Berdakin, M.; Taccone, M.; Julian, K. J.; Pino, G.; Sánchez, C. G. Disentangling the Photophysics of DNA-Stabilized Silver Nanocluster Emitters. J. Phys. Chem. C 2016, 120, 24409-24416.

(30) Brown, S. L.; Hobbie, E. K.; Tretiak, S.; Kilin, D. S. First-Principles Study of Fluorescence in Silver Nanoclusters. J. Phys. Chem. C 2017, 121, 23875-23885.

(31) Taccone, M. I.; Berdakin, M.; Pino, G. A.; Sánchez, C. G. Optical Properties and Charge Distribution in Rod-Shape DNA-Silver Cluster Emitters. Phys. Chem. Chem. Phys. 2018, 20, 22510-22516.

(32) Lisinetskaya, P. G.; Mitrić, R. Ab Initio Simulations of Light Propagation in Silver Cluster Nanostructures. Phys. Rev. B 2014, 89, 035433.

(33) Lisinetskaya, P. G.; Mitrić, R. Optimal Control of Light Propagation and Exciton Transfer in Arrays of Molecular-Like Noble-Metal Clusters. Phys. Rev. B 2015, 91, 125436. 
(34) Kondo, J.; Tada, Y.; Dairaku, T.; Hattori, Y.; Saneyoshi, H.; Ono, A.; Tanaka, Y. A Metallo-DNA Nanowire with Uninterrupted One-dimensional Silver Array. Nat. Chem. 2017, 9, 956-960.

(35) Buceta, D.; Busto, N.; Barone, G.; Leal, J. M.; Domnguez, F.; Giovanetti, L. J.; Requejo, F. G.; Garca, B.; Lpez-Quintela, M. A. Ag2 and Ag3 Clusters: Synthesis, Characterization, and Interaction with DNA. Angew. Chem. Int. Ed. 2015, 54, 7612-7616.

(36) Copp, S. M.; Schultz, D. E.; Swasey, S.; Gwinn, E. G. Atomically Precise Arrays of Fluorescent Silver Clusters: A Modular Approach for Metal Cluster Photonics on DNA Nanostructures. ACS Nano 2015, 9, 2303-2310, PMID: 25630562.

(37) Runge, E.; Gross, E. K. U. Density-Functional Theory for Time-Dependent Systems. Phys. Rev. Lett. 1984, 52, 997-1000.

(38) Lisinetskaya, P. G.; Mitrić, R. Simulation of Laser-Induced Coupled Electron-Nuclear Dynamics and Time-Resolved Harmonic Spectra in Complex Systems. Phys. Rev. A 2011, 83 .

(39) Bogh, S. A.; Cerretani, C.; Kacenauskaite, L.; Carro-Temboury, M. R.; Vosch, T. Excited-State Relaxation and Frster Resonance Energy Transfer in an Organic Fluorophore/Silver Nanocluster Dyad. ACS Omega 2017, 2, 4657-4664.

(40) Yanai, T.; Tew, D.; Handy, N. A New Hybrid Exchange-Correlation Functional Using the Coulomb-Attenuating Method (CAM-B3LYP). Chem. Phys. Lett. 2004, 393, 5157.

(41) Weigend, F.; Ahlrichs, R. Balanced Basis Sets of Split Valence, Triple Zeta Valence and Quadruple Zeta Valence Quality for H to Rn: Design and Assessment of Accuracy. Phys. Chem. Chem. Phys. 2005, 7, 3297-3305. 
(42) Frisch, M. J.; Trucks, G. W.; Schlegel, H. B.; Scuseria, G. E.; Robb, M. A.; Cheeseman, J. R.; Scalmani, G.; Barone, V.; Mennucci, B.; Petersson, G. A. Gaussian 09, Revision D.01. 2013. 